\title{
Administration of Tomato Juice or Aqueous Components of Tomato Reduces Fatigue Induced by Acute Treadmill Exercise
}

\author{
Kei Mukuta ${ }^{{ }^{*}}$, Kazuyoshi Koizumi', Eisuke F. Sato ${ }^{2,3}$, Keiichi Hiramoto ${ }^{2,3}$, Masao Hiwatari ${ }^{4}$, \\ Satoshi Sunabori ${ }^{1}$, Hiroyuki Suganuma ${ }^{1}$ \\ ${ }^{1}$ Innovation Division, KAGOME. Co. Ltd., Tochigi, Japan \\ ${ }^{2}$ Department of Biochemistry and Molecular Pathology, Osaka City University Medical School, Osaka, Japan \\ ${ }^{3}$ Faculty of Pharmacological Science, Suzuka University of Medical Science, Mie, Japan \\ ${ }^{4}$ Research Center of Clinical Medicine, International University of Health and Welfare, Tochigi, Japan \\ Email: "Kei_Mukuta@kagome.co.jp
}

Received 12 May 2016; accepted 11 June 2016; published 15 June 2016

Copyright @ 2016 by authors and Scientific Research Publishing Inc.

This work is licensed under the Creative Commons Attribution International License (CC BY).

http://creativecommons.org/licenses/by/4.0/

(c) (i)

\section{Abstract}

Physical exercise is a good means of enhancing and maintaining health. However, many people struggle to make exercise be a habit, in part because of the associated fatigue. The present study investigated the effects of tomato juice on fatigue induced by exercise in humans and mice. A study of 10 healthy men and women indicated that drinking tomato juice suppressed post-exercise increases in rated perceived exertion, a subjective fatigue indicator. In mice, a single administration of tomato juice significantly attenuated exercise-induced fatigue, as measured by locomotor activity and blood transforming growth factor $\beta$ and corticosterone levels. The active substances in tomatoes, which were among the aqueous components as of tomato juice supernatant, showed the same anti-fatigue effects as whole tomato juice. Moreover, these effects were mimicked by water supplemented with the aqueous components (i.e., sugars, organic acids, and amino acids) and were likely exerted by two or more of these components. Our results suggest that consuming tomato juice prior to or during an exercise session can reduce post-exercise fatigue, which could encourage more individuals to incorporate a regular exercise regimen into their daily routine.

\section{Keywords}

Tomato, Exercise, Fatigue

\footnotetext{
${ }^{*}$ Corresponding author.
}

How to cite this paper: Mukuta, K., Koizumi, K., Sato, E.F., Hiramoto, K., Hiwatari, M., Sunabori, S. and Suganuma, H. (2016) Administration of Tomato Juice or Aqueous Components of Tomato Reduces Fatigue Induced by Acute Treadmill Exercise. Food and Nutrition Sciences, 7, 524-532. http://dx.doi.org/10.4236/fns.2016.77054 


\section{Introduction}

Regular exercise has many health-promoting benefits, including the maintenance of physical functions [1] and prevention of metabolic syndromes [2] and various types of disease [3]-[5]. The Japanese Ministry of Health, Labour, and Welfare recommends regular exercise (>30 min at least twice a week). However, the percentage of people that habitually exercise is reportedly low: 33.8\% in men and $27.2 \%$ in women according to a 2013 survey [6]. One reason for these low rates is the negative perception of exercise as an exhausting activity. It follows that more people would adopt the habit of regular exercise if exercise-induced fatigue could be reduced.

Fatigue is caused by specific factors in muscle cells (peripheral fatigue) and diminished activation of the central nervous system (central fatigue). Peripheral fatigue associated with the depletion of energy, accumulation of metabolites such as lactate, and oxidative stress [7] [8]. Although the mechanism of central fatigue is complex, it is thought to result from an increase in central serotonin (5-HT) level and inflammatory cytokine release from muscles [7]. Consumption of certain foods is an easy way to prevent both peripheral and central fatigue; for example, carbohydrates have been suggested to delay fatigue during prolonged exercise [9] [10], and a mixture of specific branched-change amino acids (BCAAs; e.g., valine, leucine, and isoleucine) reportedly provides energy for muscles and inhibits central 5-HT release [11] [12]. Antioxidants such as vitamins C or E have also been shown to prevent oxidative stress and muscular fatigue [13] [14].

Tomato (Solanum lycopersicum) is one of the most widely consumed vegetables worldwide and contains many beneficial components, including sugars, amino acids, minerals, vitamins, and the antioxidant lycopene [14]. As such, tomato can be expected to reduce fatigue via multiple mechanisms. We investigated this possibility in the present study by evaluating the anti-fatigue effects of tomato juice in humans and mice following exercise.

\section{Materials and Methods}

\subsection{Materials}

Commercially available tomato juice (KAGOME Co., Ltd., Tochigi, Japan) and mineral water (House Foods Corp., Osaka, Japan) were used in the human study; the nutritional information is shown in Table 1. For the animal study, tomato paste (Tat Gida Sanayi AS, Istanbul, Turkey) was used to prepare tomato juice and tomato juice supernatant (hereafter referred to as supernatant) samples. Tomato juice was prepared by diluting tomato paste with distilled water to a final degree Brix of 5.3- which was the same as that of the tomato juice used in the human study - followed by centrifugation at $1660 \times \mathrm{g}$ for $10 \mathrm{~min}$ at $4^{\circ} \mathrm{C}$ to separate the serum fraction. The supernatant sample was prepared by adding the same weight of distilled water as that of the centrifuged sediment to the serum to obtain a final degree Brix of 3.9. The composition of the mixed solution of sugars, organic acids (OAs), and amino acids (AAs) was determined by high-performance liquid chromatography, and is shown in Table 2.

Table 1. Nutritional composition of experimental beverage for human study.

\begin{tabular}{|c|c|c|}
\hline & Tomato juice & Water \\
\hline Energy (kcal) & 68 & 0 \\
\hline Protein (g) & 2.2 & 0 \\
\hline Fat (g) & 0 & 0 \\
\hline Total carbohydrate (g) & 14 & 0 \\
\hline Dietary fiber (g) & 2.2 & - \\
\hline Sodium, Na (mg) & $2 \sim 36$ & 6.1 \\
\hline Calcium, Ca (mg) & 22 & 9.0 \\
\hline Potassium, K (mg) & 920 & 0.14 \\
\hline Lycopene (mg) & 34 & - \\
\hline$\beta$-carotene (mg) & 2.4 & - \\
\hline Vitamin $A(\mu g)$ & 172 & - \\
\hline
\end{tabular}

\footnotetext{
-: not measured.
} 
Table 2. Nutritional composition of experimental beverages for the animal study.

\begin{tabular}{|c|c|c|c|c|c|c|c|}
\hline & & Sugar & OA & EAA & NEAA & $\mathrm{EAA}+\mathrm{NEAA}$ & ALL \\
\hline Sugars & Glucose & 0.957 & & & & & 0.957 \\
\hline (g/100g) & Fructose & 1.256 & & & & & 1.256 \\
\hline Organic acids & Citric acid & & 0.431 & & & & 0.431 \\
\hline (g/100g) & Malic acid & & 0.044 & & & & 0.044 \\
\hline Amino acids & Thr & & & 6.654 & & 6.654 & 6.654 \\
\hline \multirow[t]{19}{*}{ (mg/100g) } & Val & & & 3.812 & & 3.812 & 3.812 \\
\hline & Met & & & 1.467 & & 1.467 & 1.467 \\
\hline & Ile & & & 2.319 & & 2.319 & 2.319 \\
\hline & Leu & & & 1.899 & & 1.899 & 1.899 \\
\hline & Phe & & & 3.549 & & 3.549 & 3.549 \\
\hline & Lys & & & 3.318 & & 3.318 & 3.318 \\
\hline & His & & & 2.801 & & 2.801 & 2.801 \\
\hline & Asp & & & & 56.757 & 56.757 & 56.757 \\
\hline & Ser & & & & 6.668 & 6.668 & 6.668 \\
\hline & Asn & & & & 24.986 & 24.986 & 24.986 \\
\hline & Glu & & & & 160.706 & 160.706 & 160.706 \\
\hline & Gly & & & & 1.219 & 1.219 & 1.219 \\
\hline & Ala & & & & 12.709 & 12.709 & 12.709 \\
\hline & Tyr & & & & 9.032 & 9.032 & 9.032 \\
\hline & Arg & & & & 1.852 & 1.852 & 1.852 \\
\hline & Pro & & & & 0.904 & 0.904 & 0.904 \\
\hline & g-ABA & & & & 24.731 & 24.731 & 24.731 \\
\hline & $\mathrm{a}-\mathrm{ABA}$ & & & & 6.982 & 6.982 & 6.982 \\
\hline & Taurine & & & & 3.034 & 3.034 & 3.034 \\
\hline
\end{tabular}

OA: organic acid; ALL: EAA + NAA + OA; EAA: essential amino acid; NAA: nonessential amino acid; g-ABA: 4-Aminobutanoic acid; a-ABA: 2Aminobutanoic acid.

\subsection{Human Study}

Ten healthy volunteers (eight men and two women ranging in age from 24 to 29) participated in a 2-day experiment to assess the effect of a single dose of tomato juice on post-exercise fatigue, with water serving as a control. The day before the each test day, subjects fasted overnight after 9 p.m. Experiments were carried out in the morning and subjects were prohibited from consuming any foods or drinks except for those being tested. Subjects wore the same clothes on each test day. There were two 30-min exercise sessions on a cycle ergometer, with a 60-min rest interval between sessions. Before the experiment, the pedal load for each subject was set so that heart rate was maintained at 130 beat per minute when pedaling at 50 revolutions per minute. Subjects were asked to drink $320 \mathrm{~g}$ of tomato juice or the same weight of mineral water immediately after the first exercise session. The experimental procedure is outlined in Figure 1. Rated perceived exertion (RPE)was recorded immediately after each exercise session according to the 15-point Borg scale, which ranges from 6 (very, very light) to 20 (very, very heavy) [15]. The study protocol was approved by the Human Research Ethics Committee of International University of Health and Welfare Hospital in accordance with the Helsinki Declaration. Prior to participation, the subjects understood and agreed with the purpose of the study and provided informed consent. 
Tomato juice or Water $\quad$ Measurement of RPE $\mathbb{R}$ Exercise

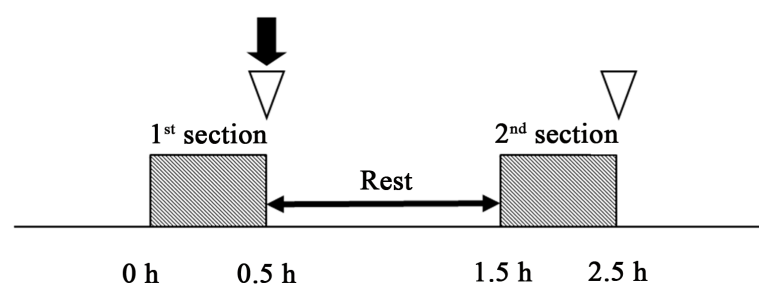

Figure 1. Experimental design of human study.

\subsection{Animal Experiments}

Male imprinting control region mice (9 weeks old) (Japan SLC, Hamamatsu, Japan) were used for experiments in accordance with the animal care regulations of Osaka City University Medical School. Mice were housed in rooms on a 12:12-h light/dark cycle with free access to aCE2 laboratory diet (CLEA Japan, Tokyo, Japan) and sterile water.

For the first experiment, animals were divided into four groups of five mice each. The control group was administered $0.3 \mathrm{ml}$ of water 60 min before the experiment but did not participate in the exercise session; the water and tomato juice groups were administered $0.3 \mathrm{ml}$ of water or tomato juice, respectively, $60 \mathrm{~min}$ before exercise; and the supernatant group was administered $0.3 \mathrm{ml}$ of supernatant $60 \mathrm{~min}$ before exercise. For the second experiment, animals were divided into eight groups of six mice each. The control group was administered $0.3 \mathrm{ml}$ of water and did not participate in the exercise session; the water group was administered $0.3 \mathrm{ml}$ of water $60 \mathrm{~min}$ before exercise; and the sugar, OA, essential (E)AA, nonessential (N)AA, EAA + NAA, and ALL (EAA + NAA + OA) groups were administered $0.3 \mathrm{ml}$ of the appropriate solutions (Table 2) $60 \mathrm{~min}$ before exercise. The experimental procedure is shown in Figure 2. Experimental samples were intragastrically administered using an animal-feeding needle.

In both experiments, animals walked on a treadmill set at an upward incline of $10^{\circ}$ (Osaka Bioscience, Osaka, Japan) with the speed incrementally increased from 0 to $25 \mathrm{~m} / \mathrm{min}$ over a $10-\mathrm{min}$ period to allow them to habituate to the device. This was carried out 3 or more days before the experiment. On the day of the experiment, each animal was orally administered $0.3 \mathrm{ml}$ of test solution $60 \mathrm{~min}$ before exercise, after which they were forced to run on the treadmill for $60 \mathrm{~min}$. The speed of the treadmill was incrementally increased from 0 to $25 \mathrm{~m} / \mathrm{min}$ in the first $10 \mathrm{~min}$ and maintained at $25 \mathrm{~m} / \mathrm{min}$ for the next $50 \mathrm{~min}$. Locomotor activity was measured immediately after the exercise session by placing the mice in a separate cage and monitoring their movement using a video camera placed above the cage. Spontaneous locomotor activity was calculated using the SMART video-tracking system (Panlab S.L., Barcelona, Spain). At $6 \mathrm{~h}$ after exercise, blood samples $(1 \mathrm{ml})$ were obtained from the heart using a syringe filled with heparin and were centrifuged at $10,000 \times \mathrm{g}$ for 10 min at $4^{\circ} \mathrm{C}$ to obtain plasma samples. Plasma levels of corticosterone and transforming growth factor (TGF)- $\beta 1$ were analyzed by enzyme-linked immunoassay (ELISA) using commercially available kits (AssayMax Corticosterone ELISA Kit, catalog no. EC3001-1; Assaypro, St. Charles, MO, USA and TGF- $\beta 1$ Emax ImmunoAssay System, catalog no. G7590; Promega, Madison, WI, USA) according to the manufacturer's instructions.

\subsection{Statistics}

Values are shown as mean $\pm \mathrm{SD}$. Differences between two groups were assessed with the Wilcoxon signed-rank test in the human study and by one-way analysis of variance followed by the Tukey-Kramer post hoc test for experiments with mice. Results were considered statistically significant at $P<0.05$.

\section{Results}

\subsection{Subjective Perception of Fatigue after Exercise in Humans}

In human experiments, mean RPE scores were significantly higher after the 2nd than after the 1st exercise session when subjects ingested water immediately after the 1stsession. On the other hand, the difference in RPE score between the 1st and 2nd exercise sessions was diminished by drinking tomato juice soon after the 1st session. 


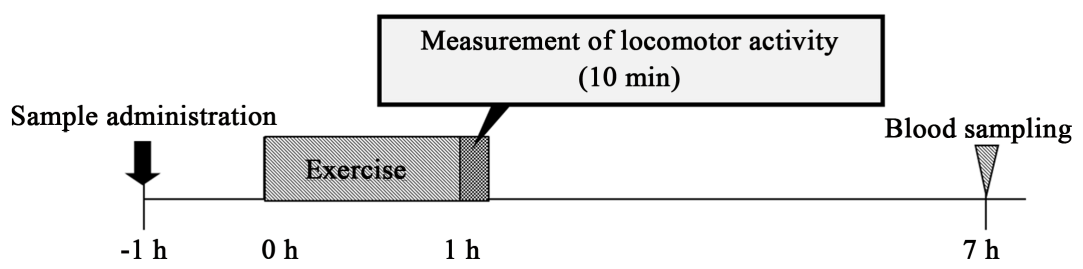

Figure 2. Experimental design for the animal study.

Scores after the 2nd session were lower in subjects drinking tomato juice as compared to water $(P=0.075$; Figure 3).

\subsection{Effects of Tomato Juice or Supernatant on Locomotor Activity and Plasma Levels of Corticosterone and TGF- $\beta$}

The effect of tomato juice or supernatant on fatigue following acute treadmill exercise in mice was determined by analyzing locomotor activity, which reflects the degree of fatigue [16]. Locomotor activity was markedly decreased in the water as compared to the nonexercise control group. Administration of tomato juice suppressed this decrease in activity, while a more potent effect was observed in mice that consumed supernatant (Figure 4(a)). Blood levels of corticosterone and TGF- $\beta$ were increased in the water group, but these were suppressed by tomato juice or supernatant (Figure 4(b) and Figure 4(c)).

\subsection{Aqueous Components in Tomato Contributing to the Alleviation of Post-Exercise Fatigue}

In order to identify the specific active ingredients contributing to the alleviation of post-exercise fatigue, we examined the effect of tomato-derived components on locomotor activity and plasma levels of corticosterone and TGF- $\beta$. Locomotor activity was found to decrease in the water, sugar, OA, EAA, NEAA, NAA, and EAA + NAA groups as compared to that in the nonexercise control group; however, the ALL group showed the same activity level as control animals (Figure 5(a)). Similar trends were observed for blood levels of corticosterone and TGF- $\beta$, with the exercise-induce increase in levels suppressed only in the ALL group (Figure 5(b) and Figure 5(c)).

\section{Discussion}

In the human experiment, ingestion of tomato juice attenuated the subjective feeling of fatigue following exercise, which was corroborated in a mouse models in which fatigue was assessed by locomotor activity and blood marker levels. This suggests that the result in humans was not a placebo effect, but reflected physiological changes induced by components of tomato juice. The same effects were observed in mice using tomato juice supernatant containing few water-insoluble components as well as a water solution composed of the same aqueous components (sugars, OAs, and AAs) as the supernatant.

Lycopene is a red lipophilic pigment found in tomatoes that is a potent, natural singlet-oxygen quencher. However, lycopene and most other lipophilic components were present in low amounts in the tomato juice supernatant (e.g., the lycopene content of the supernatant was only one-fifth that of tomato juice). Hence, the effect of tomato juice was largely attributable to the water-soluble substances in tomatoes. Indeed, supernatant suppressed the exercise-induced decline in locomotor activity to a greater extent than whole tomato juice. This may be explained by the inhibition of nutrient absorption by dietary fiber [17] [18], which could weaken the anti-fatigue effect.

In mice, only the mixed solution containing all major aqueous components of tomato (sugars, OAs, and AAs) abrogated the post-exercise decrease in locomotor activity and increase in blood markers of fatigue, indicating that anti-fatigue effects of tomato were due not to a single component but to a combination of two or more. A mixture of amino acids - especially EAAs — is known to reduce fatigue, with BCAAs contributing significantly to this phenomenon. During endurance exercise, BCAAs act as a major energy source after the depletion of carbohydrates, and increased oxidation of BCAAs triggers a gradual decrease in tricarboxylic acid cycle intermediates, resulting in fatigue [19] [20]. BCAAs are also thought to influence central fatigue, which may be me- 


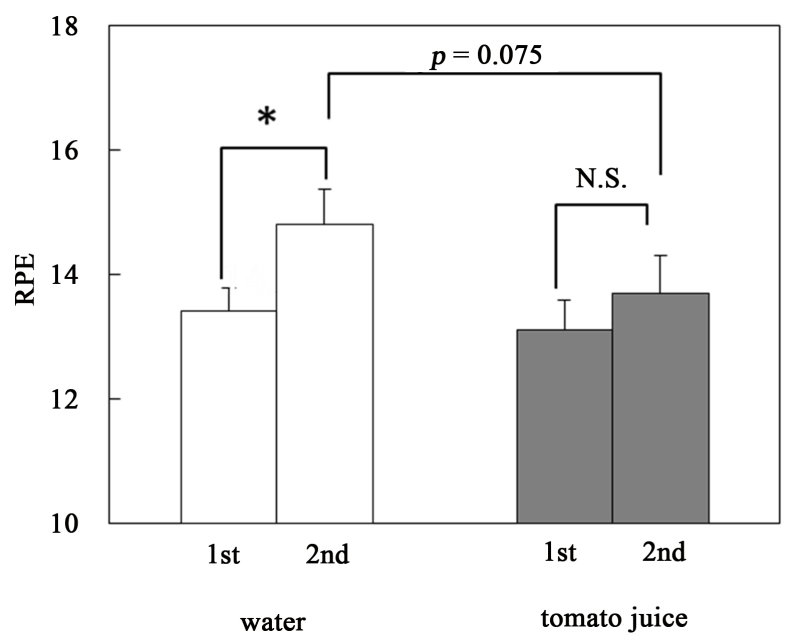

Figure 3. Changes in RPE after each exercise session. Values are expressed as mean \pm SD. ${ }^{*} P<0.05$ vs. 1 st exercise session (Wilcoxon signed-rank test).

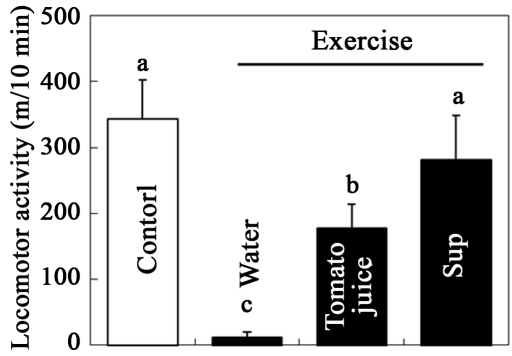

(a)

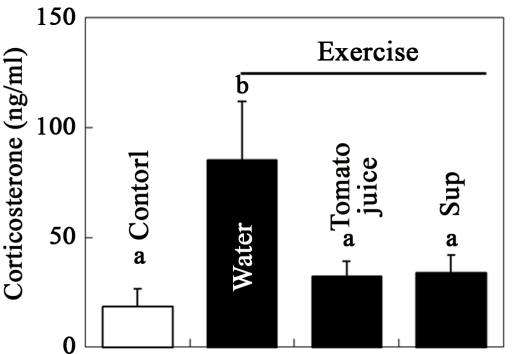

(b)

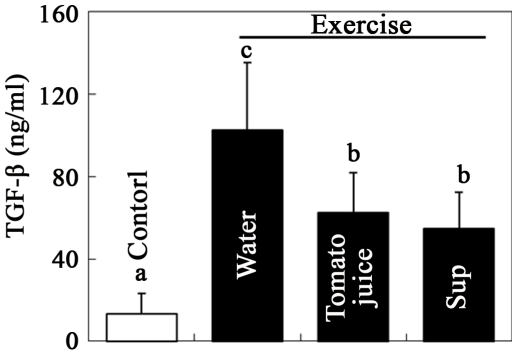

(c)

Figure 4. Changes in locomotor activity (a), blood corticosterone level (b), and blood TGF- $\beta$ level (c) after exercise. Values are expressed as mean \pm SD. Different superscript letters indicate a statistically significant difference (one-way ANOVA, post-hoc Tukey test; $P<0.05)$. Sup: tomato juice supernatant.

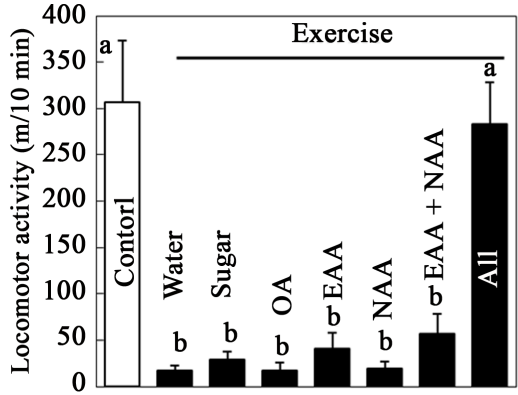

(a)

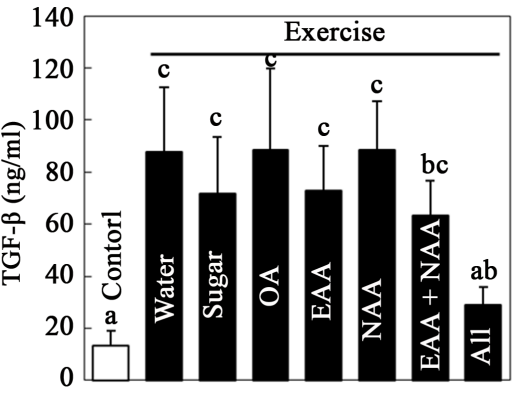

(b)

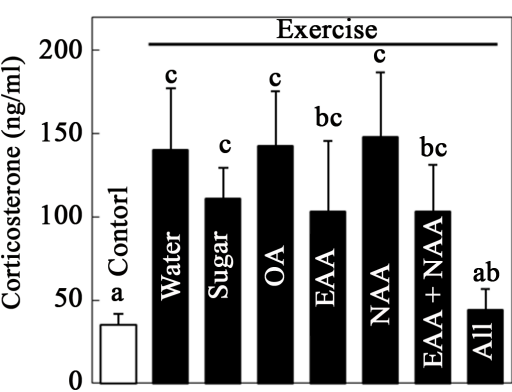

(c)

Figure 5. Changes in locomotor activity (a), blood corticosterone level (b), and blood TGF- $\beta$ level (c) after exercise. Values are expressed as mean \pm SD. Different superscript letters indicate a statistically significant difference (one-way ANOVA, post-hoc Tukey test, $P<0.05)$. ALL: sugar + OA + EAA + NEAA; EAA: essential amino acid; NAA: nonessential amino acid; OA: Organic acid.

diated by 5-HT. Brain 5-HT synthesis is stimulated in response to increased delivery to the brain of blood-borne tryptophan (Trp), an amino acid precursor of 5-HT. Trp shares the same blood-brain barrier transport mechanism as BCAAs; thus, brain 5-HT synthesis is induced by increases in the free-Trp/BCAA ratio [21]. Some studies have shown that BCAA supplementation reduces central fatigue via inhibition of brain 5-HT synthesis resulting from a decrease in blood free-Trp/BCAA ratio [22] [23]. Sugars also tend to reduce fatigue to a greater 
extent than water. Carbohydrates in the form of muscle and liver glycogen and blood glucose are the primary source of energy during exercise; one reason for declines in exercise performance is the depletion of muscle glycogen levels. Furthermore, hypoglycemia can occur during prolonged exercise, which can contribute to fatigue by limiting the supply of energy to working muscles. This can be countered by carbohydrate supplementation, which effectively improves endurance [9] [10]. In contrast, OAs had no effect of indicators of fatigue. Based on these findings, we speculate that EAAs or sugars are the components of tomato juice that are responsible for the observed anti-fatigue effects. However, the amounts were much lower than those reported to reduce fatigue [9]-[12], giving rise to the possibility of synergistic effects or the contribution of other unidentified substances.

In addition to locomotor activity, blood TGF- $\beta 1$ and corticosterone levels were measured as fatigue markers. TGF- $\beta 1$ is an anti-inflammatory cytokine that is up regulated during physical exercise [24] [25], possibly to restrict inflammatory responses to skeletal muscle damage caused by exercise [26]. Moreover, peripheral TGF- $\beta 1$ levels are higher in patients with chronic fatigue syndrome than in healthy subjects [27] [28]. On the other hand, corticosterone in rodents and cortisol in humans are glucocorticoid hormones that are secreted from the adrenal cortex via hypothalamic-pituitary-adrenal axis activation in response to psychological and physical stress [29] [30], including energy exhaustion. Glucocorticoids maintain blood glucose levels by stimulating the release of AAs from muscle and promoting gluconeogenesis is and mobilization of fat stores [31]-[33]. Corticosterone is also a peripheral marker of central 5-HT activity [34] [35]. In our experiments, changes in TGF- $\beta 1$ and corticosterone levels were correlated with decreased locomotor activity, providing evidence that these molecules are markers of fatigue. Taken together, our results indicate that TGF- $\beta 1$ and corticosterone cause post-exercise fatigue via mechanisms involving peripheral muscle inflammation, peripheral energy exertion, and the central 5-HT system.

\section{Conclusion}

We find that ingestion of tomato juice prior to exercise attenuate exercise-induced fatigue. Tomato contains many components that could prevent fatigue via synergistic and/or additive effects. This is a typical example of the benefits of a crude food containing a cocktail of nutrients. These findings are especially important given that excessive intake of refined foods have been shown to have negative effects on human health [36] [37]. Future studies will aim to identify the effective components of tomato juice and clarify their mechanisms of action.

\section{References}

[1] Manini, T.M. and Pahor, M. (2009) Physical Activity and Maintaining Physical Function in Older Adults. British Journal of Sports Medicine, 43, 28-31. http://dx.doi.org/10.1136/bjsm.2008.053736

[2] Golbidi, S., Mesdaghinia, A. and Laher, I. (2012) Exercise in the Metabolic Syndrome. Oxidative Medicine and Cellular Longevity, 2012, Article ID: 349710. http://dx.doi.org/10.1155/2012/349710

[3] Metkus Jr., T.S., Baughman, K.L. and Thompson, P.D. (2010) Exercise Prescription and Primary Prevention of Cardiovascular Disease. Circulation, 121, 2601-2604. http://dx.doi.org/10.1161/CIRCULATIONAHA.109.903377

[4] Liang, K.Y., Mintun, M.A., Fagan, A.M., Goate, A.M., Bugg, J.M., Holtzman, D.M., Morris, J.C. and Head, D. (2010) Exercise and Alzheimer's Disease Biomarkers in Cognitively Normal Older Adults. Annals of Neurology, 68, 311-318. http://dx.doi.org/10.1002/ana.22096

[5] Ahlskog, J.E. (2011) Does Vigorous Exercise Have a Neuroprotective Effect in Parkinson Disease? Neurology, 77, 288-294. http://dx.doi.org/10.1212/WNL.0b013e318225ab66

[6] Japanese Ministry of Health, Labour and Welfare (2014) National Health and Nutrition Examination Survey. http://www.mhlw.go.jp/stf/houdou/0000067890.html

[7] Finsterer, J. (2012) Biomarkers of Peripheral Muscle Fatigue during Exercise. BMC Musculoskeletal Disorders, 13, 218. http://dx.doi.org/10.1186/1471-2474-13-218

[8] Ament, W. and Verkerke, G.J. (2009) Exercise and Fatigue. Sports Medicine, 39, 389-422. http://dx.doi.org/10.2165/00007256-200939050-00005

[9] Coyle, E.F. and Coggan, A.R. (1984) Effectiveness of Carbohydrate Feeding in Delaying Fatigue during Prolonged Exercise. Sports Medicine, 1, 446-458. http://dx.doi.org/10.2165/00007256-198401060-00004

[10] Jeukendrup, A.E. (2004) Carbohydrate Intake during Exercise and Performance. Nutrition, 20, 669-677. http://dx.doi.org/10.1016/j.nut.2004.04.017 
[11] Ohtani, M., Sugita, M. and Maruyama, K. (2006) Amino Acid Mixture Improves Training Efficiency in Athletes. Journal of Nutrition, 136, 538S-543S.

[12] Shimomura, Y., Yamamoto, Y., Bajotto, G., Sato, J., Murakami, T., Shimomura, N., Kobayashi, H. and Mawatari, K. (2006) Nutraceutical Effects of Branched-Chain Amino Acids on Skeletal Muscle. Journal of Nutrition, 136, 529S-532S.

[13] Powers, S.K. and Jackson, M.J. (2008) Exercise-Induced Oxidative Stress: Cellular Mechanisms and Impact on Muscle Force Production. Physiological Reviews, 88, 1243-1276. http://dx.doi.org/10.1152/physrev.00031.2007

[14] Davies, J.N. and Hobson, G.E. (1981) The Constituents of Tomato Fruit-The Influence of Environment, Nutrition, and Genotype. CRC Critical Reviews in Food Science and Nutrition, 15, 205-280. http://dx.doi.org/10.1080/10408398109527317

[15] Borg, G.A. (1982) Psychophysical Bases of Perceived Exertion. Medicine \& Science in Sports \& Exercise, 14, 377-381. http://dx.doi.org/10.1249/00005768-198205000-00012

[16] Inoue, K., Yamazaki, H., Manabe, Y., Fukuda, C., Hanai, K. and Fushiki, T. (1999) Transforming Growth Factor-Beta Activated during Exercise in Brain Depresses Spontaneous Motor Activity of Animals. Relevance to Central Fatigue. Brain Research, 846, 145-153. http://dx.doi.org/10.1016/S0006-8993(99)01966-6

[17] Jenkins, D.J., Wolever, T.M., Leeds, A.R., Gassull, M.A., Haisman, P., Dilawari, J., Goff, D.V., Metz, G.L. and Alberti, K.G. (1978) Dietary Fibres, Fibre Analogues, and Glucose Tolerance: Importance of Viscosity. BMJ, 1, 1392-1394. http://dx.doi.org/10.1136/bmj.1.6124.1392

[18] Bolton, R.P., Heaton, K.W. and Burroughs, L.F. (1981) The Role of Dietary Fiber in Satiety, Glucose, and Insulin: Studies with Fruit and Fruit Juice. The American Journal of Clinical Nutrition, 34, 211-217.

[19] Koo, G., Woo, J., Kang, S. and Shin, K.O. (2014) Effects of Supplementation with BCAA and L-Glutamine on Blood Fatigue Factors and Cytokines in Juvenile Athletes Submitted to Maximal Intensity Rowing Performance. Journal of Physical Therapy Science, 26, 1241-1246. http://dx.doi.org/10.1589/jpts.26.1241

[20] Wagenmakers, A.J., Coakley, J.H. and Edwards, R.H. (1990) Metabolism of Branched-Chain Amino Acids and Ammonia during Exercise: Clues from McArdle’s Disease. International Journal of Sports Medicine, 11, S101-S113. http://dx.doi.org/10.1055/s-2007-1024861

[21] Davis, J.M. (1996) Carbohydrates, Branched-Chain Amino Acids and Endurance: The Central Fatigue Hypothesis. International Journal of Sport Nutrition, 5, S29-S38.

[22] Newsholme, E.A. and Blomstrand, E. (2006) Branched-Chain Amino Acids and Central Fatigue. Journal of Nutrition, 136, 274S- $276 \mathrm{~S}$

[23] Mittleman, K.D., Ricci, M.R. and Bailey, S.P. (1998) Branched-Chain Amino Acids Prolong Exercise during Heat Stress in Men and Women. Medicine \& Science in Sports \& Exercise, 30, 83-91. http://dx.doi.org/10.1097/00005768-199801000-00012

[24] Perry, C., Pick, M., Bdolach, N., Hazan-Halevi, I., Kay, S., Berr, I., Reches, A., Harishanu, Y. and Grisaru, D. (2013) Endurance Exercise Diverts the Balance between Th17 Cells and Regulatory T Cells. PLoS ONE, 8, e74722. http://dx.doi.org/10.1371/journal.pone.0074722

[25] Hamada, K., Vannier, E., Sacheck, J.M., Witsell, A.L. and Roubenoff, R. (2005) Senescence of Human Skeletal Muscle Impairs the Local Inflammatory Cytokine Response to Acute Eccentric Exercise. The FASEB Journal, 19, $264-266$.

[26] Terra, R., da Silva, S.A.G., Pinto, V.S. and Dutram, P.M.L. (2012) Effect of Exercise on the Immune System: Response, Adaptation and Cell Signaling. Revista Brasileira de Medicina do Esporte, 18, 208-214. http://dx.doi.org/10.1590/S1517-86922012000300015

[27] Patarca-Montero, R., Mark, T., Fletcher, M.A. and Klimas, N.G. (2000) Immunology of Chronic Fatigue Syndrome. Journal of Chronic Fatigue Syndrome, 6, 69-107. http://dx.doi.org/10.1300/J092v06n03_06

[28] Chao, C.C., Janoff, E.N., Hu, S.X., Thomas, K., Gallagher, M., Tsang, M. and Peterson, P.K. (1991) Altered Cytokine Release in Peripheral Blood Mononuclear Cell Cultures from Patients with the Chronic Fatigue Syndrome. Cytokine, 3, 292-298. http://dx.doi.org/10.1016/1043-4666(91)90497-2

[29] Hill, E.E., Zack, E., Battaglini, C., Viru, M., Viru, A. and Hackney, A.C. (2008) Exercise and Circulating Cortisol Levels: The Intensity Threshold Effect. Journal of Endocrinological Investigation, 31,587-591. http://dx.doi.org/10.1007/BF03345606

[30] Coleman, M.A., Garland Jr., T., Marler, C.A., Newton, S.S., Swallow, J.G. and Carter, P.A. (1998) Glucocorticoid Response to Forced Exercise in Laboratory House Mice (Mus domesticus). Physiology \& Behavior, 63, 279-285. http://dx.doi.org/10.1016/S0031-9384(97)00441-1

[31] Shephard, R.J. and Sidney, K.H. (1975) Effects of Exercise on Plasma Growth Hormone and Cortisol Levels in Human Subjects. Exercise \& Sport Sciences Reviews, 3, 1-30. http://dx.doi.org/10.1249/00003677-197500030-00004 
[32] Terjung, R. (1979) Endocrine Response to Exercise. Exercise \& Sport Sciences Reviews, 7, 153-180. http://dx.doi.org/10.1249/00003677-197900070-00007

[33] Tharp, G.D. (1975) The Role of Glucocorticoids in Exercise. Medicine \& Science in Sports, 7, 6-11. http://dx.doi.org/10.1249/00005768-197500710-00003

[34] Strachan, A.T., Leiper, J.B. and Maughan, R.J. (2004) Paroxetine Administration to Influence Human Exercise Capacity, Perceived Effort or Hormone Responses during Prolonged Exercise in a Warm Environment. Experimental Physiology, 89, 657-664. http://dx.doi.org/10.1113/expphysiol.2004.027839

[35] Van de Kar, L.D. (1997) 5-HT Receptors Involved in the Regulation of Hormone Secretion. In: Baumgarten, H.G. and Göthert, M., Eds., Serotonergic Neurons and 5-HT Receptors in the CNS, Volume 129 of Handbook of Experimental Pharmacology, Springer-Verlag, Berlin, 537-562.

[36] Isbrucker, R.A. and Burdock, G.A. (2006) Risk and Safety Assessment on the Consumption of Licorice Root (Glycyrrhiza sp.), Its Extract and Powder as a Food Ingredient, with Emphasis on the Pharmacology and Toxicology of Glycyrrhizin. Regulatory Toxicology and Pharmacology, 46, 167-192. http://dx.doi.org/10.1016/j.yrtph.2006.06.002

[37] Schmidt, M., Schmitz, H.J., Baumgart, A., Guédon, D., Netsch, M.I., Kreuter, M.H., Schmidlin, C.B. and Schrenk, D. (2005) Toxicity of Green Tea Extracts and Their Constituents in Rat Hepatocytes in Primary Culture. Food and Chemical Toxicology, 43, 307-314. http://dx.doi.org/10.1016/j.fct.2004.11.001 